\title{
Plug-Assisted Retrograde Transvenous Obliteration of Spontaneous Splenorenal Shunt for Refractory Hepatic Encephalopathy: Case Series
}

\author{
Yena Kang', Eun Jung Kim', Sang Gyune Kim', Young Seok Kim', Jae Myeong Lee², Boo Sung Kim \\ Departments of ${ }^{1}$ Internal Medicine and ${ }^{2}$ Radiology, Soonchunhyang University Bucheon Hospital, Soonchunhyang University College of Medicine, Bucheon, \\ Korea
}

Intervention treatment such as balloon retrograde or anterograde transvenous obliteration has been used for management of refractory hepatic encephalopathy as well as gastric variceal bleeding. Recently, plug-assisted retrograde transvenous obliteration without a help of balloon was newly developed to treat these patients. Here, we report three cases suffering refractory hepatic encephalopathy who were treated with this new technique.

Keywords: Hepatic encephalopathy; Balloon occlusion; Portosystemic shunt, surgical

\section{INTRODUCTION}

Hepatic encephalopathy (HE) is one of most dreadful complications in patients with decompensated liver cirrhosis. Spontaneous portosystemic shunt mainly contributes to this condition where ammonia directly makes an adverse effect on brain due to shift of blood flow from the liver. Balloon retrograde transvenous obliteration (BRTO) has been used to block the blood flow passing through a portosystemic bypass such as splenorenal or gastrorenal shunt. This procedure can help control bleeding of gastric varices and manage refractory HE. The major drawback of BRTO usually comes from the toxicity of ethanolamine oleate or balloon rupture during procedure. Recently, a vascular plug and gelatin spongeassisted retrograde transvenous obliteration (PARTO) is applied in place of BRTO to overcome adverse events. In this modified procedure, vascular plug is deployed in a distal outflow of portosystemic shunt instead of balloon, and then gelfoam is infused to occlude the shunt. In this article, we are to present the cases with refractory HE who were successfully treated with PARTO and give a review of its effectiveness for management of $\mathrm{HE}$.

\section{CASE REPORTS}

\section{Case 1}

A 75-year-old man with decompensated liver cirrhosis caused by chronic alcohol drinking, admitted to Soonchunhyang University Bucheon Hospital several times due to HE. Symptoms included slow speech, loss of orientation, drowsy mentality and insomnia. Though he already had been prescribed lactulose $120 \mathrm{~mL} /$ day and rifaximine $1,200 \mathrm{mg} /$ day in outpatient clinic, flapping tremor and disorientation were sustained and he had many ups and downs on encephalopathy. There was no risk factor associated with worsening of symptoms out there. The patient had Child-Pugh class C and Model for End Stage Liver Disease (MELD) score of 15.

He received the total gastrectomy operation due to early gastric cancer 13 years ago. So there was no gastric varices, but perisplenic, paravertebral and hemorrhoidal collateral vessels and gastrorenal shunt were prominent on computed tomography (CT) scans (Fig. 1).

We explained the PARTO for the prevention of HE to the patient and his family, and they agreed to perform this procedure. It was done by an intervention radiologist in our hospital. A careful selection and measurement of diameter of gastrorenal shunt was 

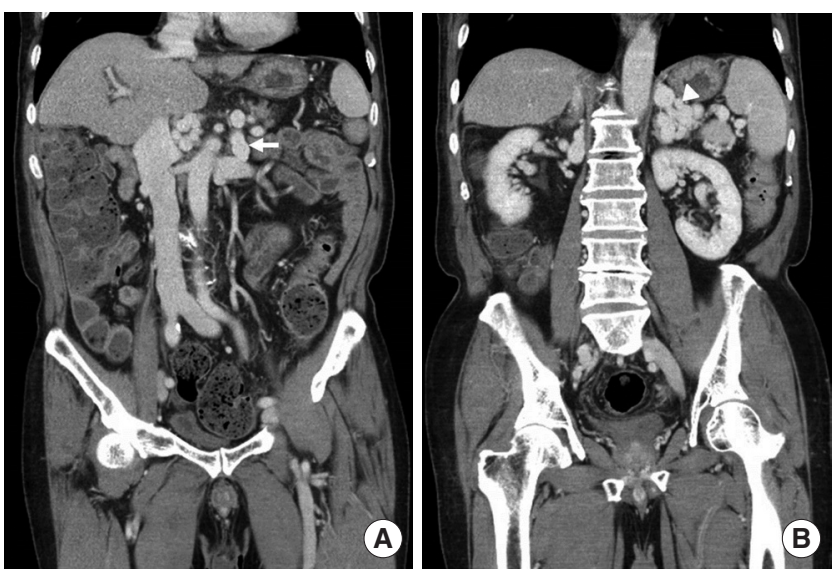

Fig. 1. Coronal reformatted abdomen computed tomography of case 1. (A) Large shunt vessel to approach the gastric varices is seen on enhanced abdomen computed tomography scan (arrow). (B) Perisplenic collateral vessels are prominent above left kidney (arrowhead).

Table 1. Pre- and post-procedural ammonia levels

\begin{tabular}{lcc}
\hline & \multicolumn{2}{c}{ Ammonia levels $(\mu \mathrm{g} / \mathrm{dL})$} \\
\cline { 2 - 3 } Patient & Pre-procedure & $\begin{array}{c}\text { Post-procedure } \\
\text { (the value of after } 3 \text { or } 4 \text { weeks) }\end{array}$ \\
\hline Case 1 & 232 & 79 \\
Case 2 & 226 & 57 \\
Case 3 & 340 & 80 \\
\hline
\end{tabular}

Post-procedural ammonia levels of all patients are dropped remarkably and mean decline was $194 \mu \mathrm{g} / \mathrm{dL}$. Post-procedural level of first patient was checked after 3 weeks from plug-assisted retrograde transvenous obliteration.

followed by a vascular plug placement and gelfoam injection via right femoral vein (Fig. 2).

After the procedure, the patient felt a fever and chilling for a while, so we used prophylactic antibiotics during 3 days and this symptom was subsided. The level of plasma ammonia before BRTO was $232 \mu \mathrm{g} / \mathrm{dL}$ (normal value, 15 to $45 \mu \mathrm{g} / \mathrm{dL}$ ). After 4 weeks, the level was dropped to $79 \mu \mathrm{g} / \mathrm{dL}$ (Table 1). Levels of total bilirubin, serum albumin, and prothrombin time have been shown similar level with before and after procedure. The patient has never experienced HE during next 3 months. Furthermore, follow-up CT scans showed almost obliterated splenorenal shunt and collateral vessels, but aggravated ascites is seen (Fig. 3).

\section{Case 2}

A 59-year-old woman under treatment for alcoholism in nursing hospital, was transferred to Soonchunhyang University Bucheon Hospital due to confused mental state. She was already diagnosed with alcoholic liver cirrhosis. Child-Pugh class was C,
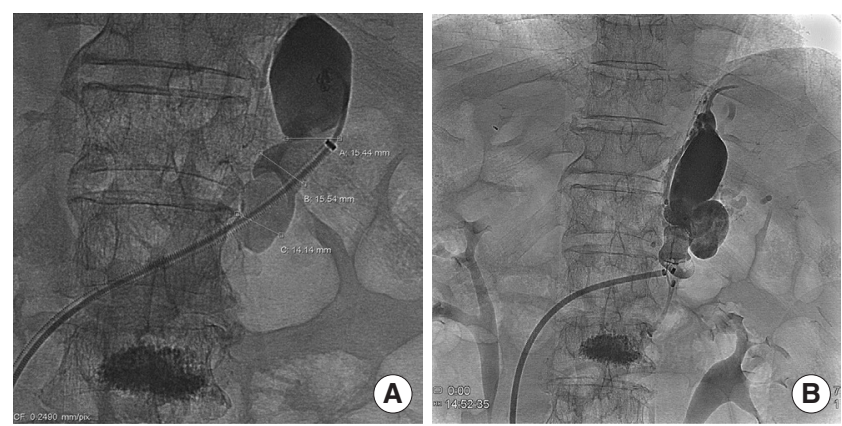

Fig. 2. Intervention radiologist selected the vessel carefully and measured the diameter of gastrorenal shunt. (A) Largest diameter of shunt was $15.54 \mathrm{~mm}$. (B) Gelfoam was injected after vascular plug placement.
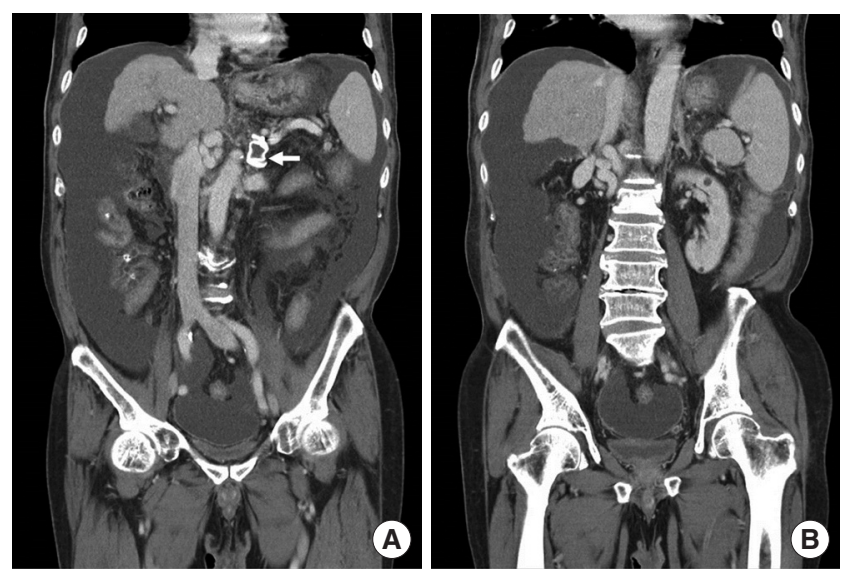

Fig. 3. Coronal reformatted abdomen computed tomography after procedure of case 1. (A) Follow-up abdomen computed tomography scans taken after 1 month from procedure showed almost obliterated splenorenal shunt (arrow) and (B) perisplenic collateral vessels. But amount of ascites was increased.

and MELD score was 22 . Her family said she had experienced a few episodes of HE. Plasma ammonia level was $226 \mu \mathrm{g} / \mathrm{dL}$ at admission. The patient was taken care of in intensive care unit and her consciousness was recovered by lactulose enema. On her CT scan we found many collateral vessels and prominent splenorenal shunt. All her family members didn't agree to our suggestion that she had to have liver transplantation. We planned PARTO for prevention of recurrent $\mathrm{HE}$. It was done successfully by vascular plug and gelfoam without any complication.

After 1 month from procedure, plasma ammonia level was dropped to $57 \mu \mathrm{g} / \mathrm{dL}$ at outpatient clinic (Table 1). Following 3 months after the PARTO, no complications or symptoms related to procedure were developed and there is no significant change with level of ammonia (Fig. 4).

\section{Case 3}

A 59-year-old man with a past history of hepatitis B inactive 


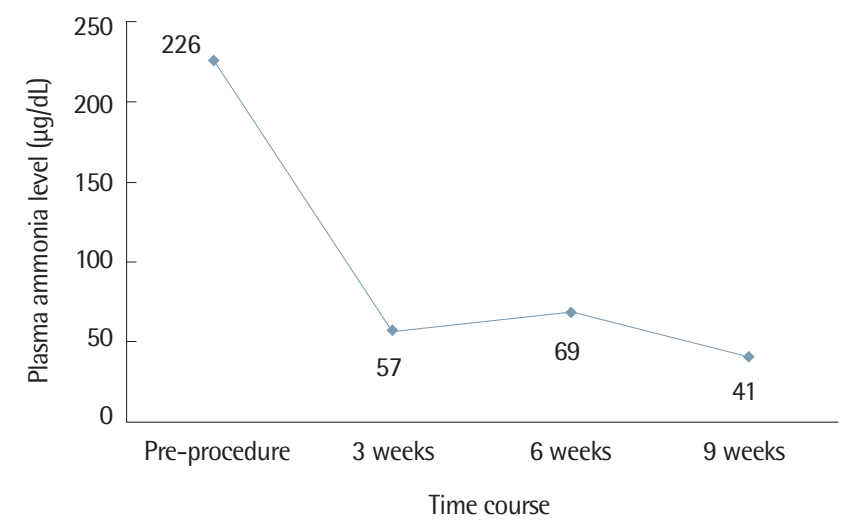

Fig. 4. Change of plasma ammonia levels of case 2. Serial check-up of plasma ammonia level was done at outpatient clinic. The level of ammonia has decreased significantly after procedure and tends to be stable.

carrier and alcoholic cirrhosis was hospitalized due to drowsy mental state sustained during a week. The patient had ChildPugh class C and MELD score of 16.

When he arrived at hospital, his mental status was confused and disoriented. Plasma ammonia level was $340 \mu \mathrm{g} / \mathrm{dL}$. After treated with lactulose enema and fluid therapy at intensive care unit for two days, he came to his senses. The abdomen CT scans showed the splenorenal shunt and many other collateral vessels. Actually he had no close relative to support him. PARTO was performed by the same intervention radiologist through gastrorenal shunt after puncture of right femoral vein. There were two shunt vessels. A larger one was embolized with vascular plug and smaller one was occluded by coil. Then, gelfoam was infused to block these shunt vessels. The patient felt comfortable and no complication happened to him during the stay of hospital.

During 3 months follow-up period, encephalopathy did not recur to him and plasma ammonia level was checked at $80 \mu \mathrm{g} / \mathrm{dL}$ at outpatient clinic (Table 1). But his abdomen looked distended probably due to development of ascites. He was strongly asked to keep alcohol abstinence and use of diuretics.

\section{DISCUSSION}

$\mathrm{HE}$ is defined as a neuropsychiatric manifestation of chronic and acute liver disease with disturbances of psychomotor, cognitive, emotional, behavioral, and fine motor functions. Shunting of portal blood flow into the systemic circulation makes ammonia delivered to brain. The unremoved ammonia plays main role in the pathogenesis of HE [1].
The conventional management of HE has been focused on reduction of ammonia level in the body, which includes restriction of dietary proteins, administration of lactulose, or probiotics. Lactulose can be administered via oral or by enema. It can remove ammoniagenic substrates from the intestinal lumen by the osmotic cathartic action. Probiotics (e.g., rifaximin) modulate gut flora and reduce production of ammonia. However, they are not likely to be effective to prevent production and absorption of ammonia [2].

The patients with advanced liver cirrhosis have multiple portosystemic shunts. Thus obliteration of shunt could be an effective way to relieve and prevent HE symptoms [3-7], especially, in case gastric varix is large enough to be approached because it is commonly connected with gastrorenal shunt. There are two mechanisms of improvement of HE through BRTO. One is that the obliteration of the shunting into the systemic circulation can reduce the systemic outflow of neurotoxins, and the other is that the obliteration of hepatofugal shunts causes reversal of blood flow, thus improving blood flow towards the liver.

BRTO procedure starts with inserting a balloon catheter into an outflow shunt (gastric-renal or gastric-vena caval inferior) via access of the femoral or internal jugular vein. Then, Inflated balloon block the blood flow and sclerosing agent is injected in a retrograde manner [8]. This method has been performed for last decades.

But there are several raising concerns about complications following this procedure. Ethanolamine oleate, which is most commonly used sclerosant in BRTO, has complications including severe renal dysfunction, pulmonary edema, cardiogenic shock, disseminated intravascular coagulation, and anaphylactic reaction. Furthermore balloon rupture during the procedure can result in symptomatic pulmonary embolism [9].

In recent years, there has been an advance in sclerosing agent and plugging. PARTO is a modified method of BRTO which utilizes gelfoam and vascular plug instead of indwelling balloon in shunt. Therefore it could be done without serious complications above mentioned. This method is also applied to patients with HE. Gwon et al. [9] reported that the successful result of PARTO with 20 patients. In this study, symptoms of encephalopathy were resolved in all patients with overt $\mathrm{HE}(\mathrm{n}=7)$, and no other complications and recurrence of $\mathrm{HE}$ were seen during mean follow-up of 422 days.

Our report showed a therapeutic effect of PARTO in selected patients with HE. There are some limitations in our case reports. First, follow-up period is only 3 months during which it is not 
Kang Y, et al. • Plug-Assisted Retrograde Transvenous Obliteration for Hepatic Encephalopathy: Case Series

enough to look for a long term effect or complications of PARTO. Shunt occlusion is known to exacerbate the ascites or esophageal varices [10]. In our cases, aggravated ascites was occurred in 2 patients. So we consider that BRTO is not suitable for patients with uncontrolled ascites. And worsening of esophageal varices was not seen. But it is too short to proven this complication. Therefore a longer follow-up period is required in order to prove its safety and the effectiveness of preventing encephalopathy. Another limitation of this study is that we didn't have a quantifiable measurement of symptom improvement except for the reduced serum ammonia level. But it shows the meaningful value to reflect the relief of encephalopathy.

We report here that PARTO is a potential alternative of BRTO in the setting of refractory $\mathrm{HE}$ with a fewer complications. More clinical trials are needed to compare the safety and efficacy between two procedures.

\section{REFERENCES}

1. Haussinger D, Schliess F. Pathogenetic mechanisms of hepatic encephalopathy. Gut 2008;57:1156-65.

2. Riordan SM, Williams R. Treatment of hepatic encephalopathy. N Engl J Med 1997;337:473-9.

3. Kato T, Uematsu T, Nishigaki Y, Sugihara J, Tomita E, Moriwaki H. Ther- apeutic effect of balloon-occluded retrograde transvenous obliteration on portal-systemic encephalopathy in patients with liver cirrhosis. Intern Med 2001;40:688-91.

4. Fukuda T, Hirota S, Sugimura K. Long-term results of balloon-occluded retrograde transvenous obliteration for the treatment of gastric varices and hepatic encephalopathy. J Vasc Interv Radiol 2001;12:327-36.

5. Numata K, Tanaka K, Kiba T, Saito S, Shirato K, Kitamura T, et al. Use of balloon-occluded retrograde transvenous obliteration with ethanolamine oleate for the treatment of hepatic encephalopathy in a cirrhotic patient with a large spontaneous splenorenal shunt. J Gastroenterol 1998;33:4247.

6. An J, Kim KW, Han S, Lee J, Lim YS. Improvement in survival associated with embolisation of spontaneous portosystemic shunt in patients with recurrent hepatic encephalopathy. Aliment Pharmacol Ther 2014;39: 1418-26.

7. Mukund A, Rajesh S, Arora A, Patidar Y, Jain D, Sarin SK. Efficacy of balloon-occluded retrograde transvenous obliteration of large spontaneous lienorenal shunt in patients with severe recurrent hepatic encephalopathy with foam sclerotherapy: initial experience. J Vasc Interv Radiol 2012;23: 1200-6.

8. Kanagawa H, Mima S, Kouyama H, Gotoh K, Uchida T, Okuda K. Treatment of gastric fundal varices by balloon-occluded retrograde transvenous obliteration. J Gastroenterol Hepatol 1996;11:51-8.

9. Gwon DI, Ko GY, Yoon HK, Sung KB, Kim JH, Shin JH, et al. Gastric varices and hepatic encephalopathy: treatment with vascular plug and gelatin sponge-assisted retrograde transvenous obliteration--a primary report. Radiology 2013;268:281-7.

10. Inoue M, Tanaka T, Nakagawa H, Yoshioka T, Kichikawa K. Splenic vein embolization using coil anchors and prophylactic occlusion of a hepatofugal collateral for hepatic encephalopathy due to splenorenal shunt: technical note and literature review. Case Rep Radiol 2013;2013:160653. 